\title{
Optical Properties of Poly(para-phenylene Vinylene) and Single-Walled Carbon Nanotube Composite Films: Effects of Conversion Temperature, Precursor Dilution, and Nanotube Concentrations
}

\author{
F. Massuyeau, H. Aarab, L. Mihut, S. Lefrant, E. Faulques,* and J. Wéry \\ Institut des Matériaux Jean Rouxel, Nantes Atlantic Universities 2 rue de la Houssinière, BP32229, \\ F-44322 Nantes, France \\ E. Mulazzi and R. Perego \\ Dipartimento di Fisica, Universitá degli Studi di Milano and European Theoretical Spectroscopy Facility \\ (ETSF), CNISM-CNR-INFM, Via Celoria, 16, 20133 Milano, Italy
}

Received: June 15, 2007

\begin{abstract}
Optical absorption, photoluminescence, and Raman scattering spectra of poly(para-phenylene vinylene) (PPV) and single-walled carbon nanotube (SWNT) composite films are investigated at room temperature. Samples have been prepared at different precursor conversion temperatures, $T_{\mathrm{c}},\left(300,180\right.$, and $\left.120^{\circ} \mathrm{C}\right)$ and with SWNT mass concentrations from $x=0 \%$ up to $64 \%$. In each sample, we observe drastic changes in all optical absorption spectra of PPV and composite films. In particular, after conversion at $T_{\mathrm{c}}=120^{\circ} \mathrm{C}$, PPV samples exhibit photoluminescence (PL) with a new feature at about $2.55 \mathrm{eV}$ together with less-intense ones at about 2.37 and $2.20 \mathrm{eV}$, respectively. The most-intense at $2.55 \mathrm{eV}$ is due to a radiative recombination on the shorter conjugated segments and interpreted from a theoretical model based on a distribution of conjugated lengths. This distribution, which allows an assignment of all PL peaks, is also able to explain all experimental data including Raman scattering and optical absorption spectra in a given sample. Also, further changes in PL and optical absorption spectra are observed by increasing the SWNT concentration in composite films converted at the same temperature. We have also investigated the effect of the dilution of the precursor polymer solution. From the theoretical analysis of the optical absorption, PL, and resonance Raman spectra, we show that PPV samples are characterized by a decrease of the effective conjugation lengths when the precursor dilution increases. All experimental data are explained well with a bimodal distribution model reflecting an effective inhomogeneity in the polymer as suggested already from morphological pictures issued in particular from X-ray data.
\end{abstract}

\section{Introduction}

Conjugated polymers are of large interest in novel optical and electro-optical devices. In particular, poly(para-phenylene vinylene) (PPV) and its derivatives are among the mostpromising organic materials for flat displays and a great deal of efforts have been directed toward the improvement of their performances through chemical processes. ${ }^{1} \mathrm{PPV}$ is the prototype of electroluminescent conjugated polymers that has been studied extensively. ${ }^{2,3}$ It is insoluble, and it is usually prepared from a precursor polyelectrolyte polymer converted either thermally and/or by UV irradiation. ${ }^{4}$ The optical properties of PPV samples depend not only on the choice of the chemical group attached to the precursor polymer ${ }^{5-7}$ but also on the nature of the solvent, ${ }^{8}$ solvent concentration, ${ }^{9}$ and the storage conditions. Then it has been shown that the conversion temperature $\left(T_{\mathrm{c}}\right)$ of the precursor polymer solution is $300{ }^{\circ} \mathrm{C}$ leading to the so-called standard PPV with optimized optical properties. Furthermore, the photoluminescence intensity of PPV has been reported to depend on the heating rate and the environment gas. ${ }^{10}$ In addition, when the conversion temperature is lowered typically between 180 and $120{ }^{\circ} \mathrm{C}$, the precursor polymer is not

* Corresponding author. E-mail: eric.faulques@cnrs-imn.fr; tel: +33240373977; fax: +33-240373991. completely converted into standard PPV, but few studies ${ }^{11-13}$ were carried out on the optical property changes as a consequence of the different $T_{\mathrm{c}}$ values. The absorption data show that the decrease of the conversion temperature leads to PPV samples characterized by shorter effective conjugation lengths because of a partial conversion of the polyelectrolyte polymer precursor. Alternatively, in composite samples, ${ }^{14}$ the increase of concentration $x$ of single-walled carbon nanotubes (SWNTs) in the precursor solution yields samples with shorter conjugated segments with respect to standard PPV. This has been proven by investigating the optical properties (optical absorption, photoluminescence, and Raman scattering) of such systems. ${ }^{14,15}$

In this paper, we report an experimental and theoretical investigation of the optical properties of both PPV and SWNTPPV composite films obtained from conversion temperatures at 120,180 , and $300{ }^{\circ} \mathrm{C}$, respectively, and for different concentrations $x$ of SWNTs. We show that dramatic changes in the PPV optical properties occur when the preparation conditions are varied.

We have also prepared thin PPV films converted at $T_{\mathrm{c}}=$ $300{ }^{\circ} \mathrm{C}$ with different polymer precursor dilutions. We find that Raman scattering and photoluminescence (PL) spectra recorded from these samples present changes in the relative intensities of the main features. These modifications are similar to those 
already recorded in samples prepared by increasing the SWNT concentration $x$ and/or decreasing the conversion temperature. In addition, transient PL experiments in the picosecond time range are shown for all samples. Experimental data are interpreted in the frame of the model based on the bimodal distribution of PPV conjugation effective lengths. This model, which is able to reproduce the different experimental spectra by using the same set of parameters, is also supported by morphological pictures deduced from PL (see ref 16) and X-ray data. ${ }^{17,18}$

\section{Experimental Details}

Composite films are prepared from a mixture of the precursor polymer diluted in ethanol and SWNTs at different mass concentrations dispersed by sonication. The obtained solutions are deposited onto silica or silicium substrates under a nitrogen flow. Beforehand, all substrates were cleaned in ultrasonical bath with deionized water and ethanol.

The PPV precursor polymer with tetra-hydrothiophenium groups is synthesized in our laboratory via a standard procedure as described elsewhere. ${ }^{19}$ The powder of SWNTs used to prepare homogeneous and stable solutions was provided by Aldrich. Then, composite films obtained after evaporation of the polar solvent are introduced in an oven and converted at different temperatures $\left(120,180\right.$, and $\left.300^{\circ} \mathrm{C}\right)$ under dynamic secondary vacuum for a duration of $6 \mathrm{~h}$. The thickness of the composite films is typically $\sim 200 \mathrm{~nm}$ as determined by profilometry. To study the influence of the precursor dilution, we have prepared PPV films starting from a solution in which the polymer mass concentration is $2.5 \mathrm{mg} / \mathrm{mL}$. We have obtained three different precursor polymer diluted solutions, respectively, $0.625,0.25$, and $0.125 \mathrm{mg} / \mathrm{mL}$, which correspond to a precursor solution mass ratio of $1: 4,1: 10$, and 1:20 with respect to the starting solution. We have deposited by drop casting the same volume of each solution on different substrates and films have been heated at $300{ }^{\circ} \mathrm{C}$ under vacuum.

Optical absorption spectra are recorded by using a Varian Cary $5 \mathrm{UV}$-visible-near-infrared spectrometer in the $1.8 \mathrm{eV}-$ $3.6 \mathrm{eV}$ range. Steady-state photoluminescence spectra are collected from the front-face geometry of the samples with a Jobin-Yvon Fluorolog spectrometer using a Xenon lamp (500 $\mathrm{W})$ as an excitation source. For time-resolved PL experiments, we use a laser system generating $30-\mathrm{fs}$ pulses at $1 \mathrm{kHz}$. Emission spectra are collected for the excitation energy $E_{\text {exc }}=3.1 \mathrm{eV}$ (400 nm). The pump energy density is set at $3.75 \mathrm{~nJ} / \mathrm{pulse}$. The transient signals are sent to a monochromator and detected with a streak camera of temporal resolution $<20$ ps. Raman scattering spectra are recorded by using an excitation laser wavelength of $1064 \mathrm{~nm}$ on a Fourier-transform Raman spectrometer Brucker RFS 100. Otherwise, resonant Raman scattering spectra are recorded for a $90^{\circ}$ scattering geometry with the 363-nm laser wavelength issued from an $\mathrm{Ar}^{+}$laser, on a multichannel JobinYvon T64000 spectrometer connected to a CCD detector. In both cases, the spectral resolution of the scattered light was 4 $\mathrm{cm}^{-1}$. Infrared absorption measurements are recorded by using a Nicolet FTIR interferometer 20 SXC with an energy resolution of $4 \mathrm{~cm}^{-1}$. X-ray diffraction patterns are measured with a diffractometer equipped with a curved position-sensitive detector INEL CPS120 allowing to us to collect simultaneously the signal in the $120^{\circ} 2 \theta$ range. The wavelength of the incident beam is $1.542 \AA$. The diffraction plane is vertical and the sample film area is horizontal. Because of the very small thickness of the film, a grazing incidence is used to increase the amount of matter for the incident beam. This last configuration may introduce

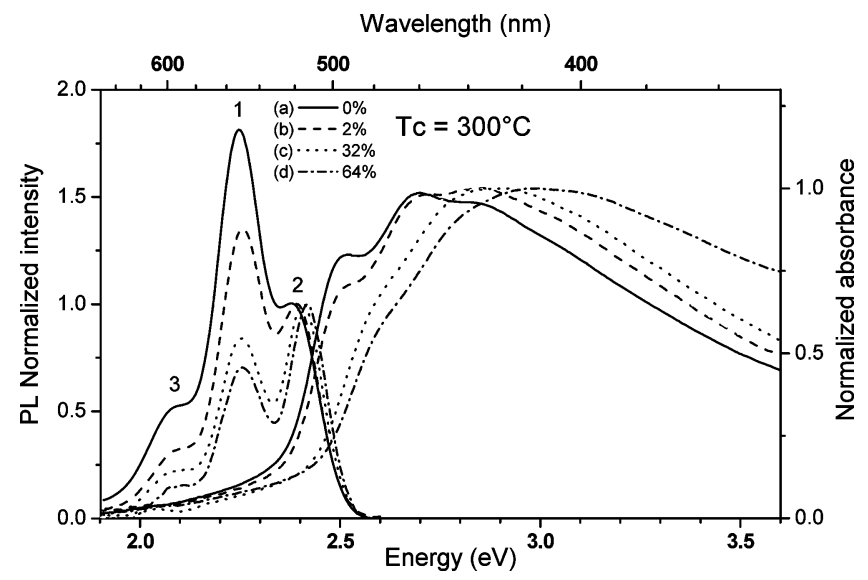

Figure 1. Room-temperature optical absorption and photoluminescence spectra of PPV-SWNT composites converted at $T_{\mathrm{c}}=300{ }^{\circ} \mathrm{C}$ for an exciting energy $\mathrm{E}_{\mathrm{exc}}=3.1 \mathrm{eV}$ for different SWNT mass percentages $x$. (a) $x=0 \%$, (b) $x=2 \%$, (c) $x=32 \%$, and (d) $x=64 \%$. Absorption curves have been normalized to the maximum intensity of peak at 2.7 $\mathrm{eV}$ of curve a. PL curves have been normalized to the intensity of peak 2 (at $\sim 2.4 \mathrm{eV}$ ), respectively.

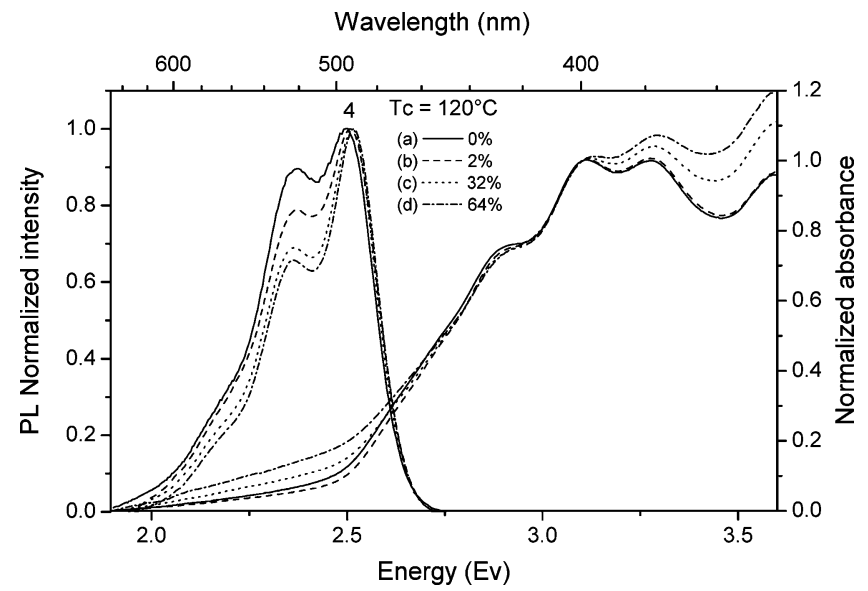

Figure 2. Room-temperature optical absorption and photoluminescence spectra recorded on PPV-SWNT composites composites converted at $T_{\mathrm{c}}=120^{\circ} \mathrm{C}$ for an exciting energy $\mathrm{E}_{\mathrm{exc}}=3.1 \mathrm{eV}$ and for different SWNT mass percentages $x$. (a) $x=0 \%$, (b) $x=2 \%$, (c) $x=32 \%$, and (d) $x=64 \%$. Absorption curves have been normalized to the maximum intensity of peak at $3.2 \mathrm{eV}$ of curve a. PL curves have been normalized to the intensity of peak 2 (at $\sim 2.4 \mathrm{eV}$ ), respectively.

some errors in the peak positions of the diffracted pattern, which, nevertheless, remain negligible with respect to the very broad peaks recorded in PPV. Another consequence of the very small sample volume is that parasitic signals are relatively large. They arise from air surrounding the sample and from the silicon substrate. All data are corrected for these effects.

All of our experimental data were obtained in ambient air at room temperature and at $87 \mathrm{~K}$ under vacuum when indicated.

\section{Results}

3.1. Effect of Conversion Temperature and of SWNT Concentration on Absorption and PL Spectra. Figures $1 \mathrm{a}-\mathrm{d}$ and $2 \mathrm{a}-\mathrm{d}$ show optical absorption and PL spectra of composite films with different SWNT $x$ concentration as indicated and at two conversion temperatures $T_{\mathrm{c}}=300$ and $120^{\circ} \mathrm{C}$, respectively. This choice of $T_{\mathrm{c}}$ is relevant because it corresponds to different stages of the thermal conversion process identified previously by thermal measurements..$^{20,21}$

On the right side of Figures $1 \mathrm{a}-\mathrm{d}$ and $2 \mathrm{a}-\mathrm{d}$, we show the optical absorption spectra of composite films, at the conversion 
temperatures $T_{\mathrm{c}}$ as indicated. They are characterized by broad bands that exhibit dramatic changes as function of the SWNT concentration $x$ in the precursor solution, as well as of the conversion temperature. This is consistent with results found in the literature. ${ }^{13,14,22-24}$ In Figure 1a, the main band, attributed to the $\pi \rightarrow \pi^{*}$ electronic transition, is structureless and redshifted compared to that of Figure $2 \mathrm{a}$. In Figure $1 \mathrm{~b}-\mathrm{d}$, the relative intensity of the absorption above $2.9 \mathrm{eV}$ increases, as discussed already in ref 14 . For the PPV film partially converted at $120^{\circ} \mathrm{C}(x=0 \%)$, in Figure 2 a the absorption curve is wellstructured and exhibits several peaks at 2.9, 3.11, 3.28, 3.65 $\mathrm{eV}$. They are attributed to the electronic transitions of short conjugated segments. In Figure $2 \mathrm{c}$ and $\mathrm{d}$, the relative intensity of all the peaks above $3.2 \mathrm{eV}$ increase for $x$ increasing.

From the experimental data presented above, the conversion temperature dependence clearly suggests that the effective conjugation length in PPV films increases when $T_{\mathrm{c}}$ increases. Alternatively, the SWNT concentration variation may indicate that the complete conversion of PPV in the composite films is progressively prevented by increasing $x$ from $0 \%$ to $64 \%$. This fact explains the blue-shift of the main absorption band.

On the left side of Figures $1 \mathrm{a}-\mathrm{d}$ and $2 \mathrm{a}-\mathrm{d}$, we present the PL experimental data of the composite films converted at $T_{\mathrm{c}}=$ $300{ }^{\circ} \mathrm{C}$ and $120{ }^{\circ} \mathrm{C}$ for different SWNT concentrations $x$ as indicated. The PL spectrum for $T_{\mathrm{c}}=300{ }^{\circ} \mathrm{C}$ shown in Figure 1a exhibits two main peaks located, respectively, at about 2.25 $\mathrm{eV}$ (peak 1) and $2.40 \mathrm{eV}$ (peak 2) with a small feature at 2.10 $\mathrm{eV}$ (peak 3). By increasing the SWNT concentration $x$, the relative intensity of peak 1 to peak 2 changes drastically. While at $x=0 \%$ (Figure 1a) peak 1 is the most intense feature, peak 2 becomes the dominant one at $x=64 \%$ (Figure 1d).

The PL spectrum of the $x=0 \%$ sample obtained at $T_{\mathrm{c}}=$ $120{ }^{\circ} \mathrm{C}$ shown in Figure 2a exhibits two main peaks, respectively, located at 2.37 and $2.55 \mathrm{eV}$ (peak 4) and a bump located around $2.23 \mathrm{eV}$. The peak at $\sim 2.37 \mathrm{eV}$ and the large bump at $\sim 2.23 \mathrm{eV}$ are almost at the same energy as that of peaks 2 and 1 , respectively. As the SWNT concentration $x$ increases in the precursor polymer, the ratio of relative intensity of peak 4 with respect to that of peak 2 becomes larger. One remarks that the relative intensity of peak 1 with respect to that of peak 2 increases with the conversion temperature. Also, from Figures 1 and 2, we note a major change in the spectra when the conversion temperature decreases. In fact, a new peak appears at $2.55 \mathrm{eV}$ in the spectra of Figures 2 that was not present in the spectra of Figure 1. This feature can be correlated with an average shortening of the conjugated segments as a consequence of a lower $T_{\mathrm{c}}$. This is a signature of the weight increase of the short segments distribution in the samples when $T_{\mathrm{c}}$ decreases. ${ }^{25}$ PL spectra for composite films obtained at $T_{\mathrm{c}}=180{ }^{\circ} \mathrm{C}$ (not shown here) are characterized by two main peaks at 2.45 and $2.31 \mathrm{eV}$, respectively, and a small bump at $2.15 \mathrm{eV}$. The energy values of these peaks are intermediate between those recorded from samples obtained at $T_{\mathrm{c}}=300{ }^{\circ} \mathrm{C}$ and $T_{\mathrm{c}}=120{ }^{\circ} \mathrm{C}$.

3.2. Effect of the Dilution of the Precursor Solution on Absorption and PL Spectra. We present in Figure 3 optical absorption and PL spectra of PPV samples obtained for three different dilutions of the precursor polymer solution: (1:4) low, (1:10) medium, and (1:20) high. In Figure $3 a-c$ (right side), we show optical absorption curves of PPV films obtained at $T_{\mathrm{c}}$ $=300{ }^{\circ} \mathrm{C}$ for the different dilutions as indicated. We observe that all spectra are similar and that there is no evolution of the optical absorption onset at $E_{0} \approx 2.35 \mathrm{eV}$, whereas the major modification is related to the absorbance intensity, which decreases by increasing dilution.

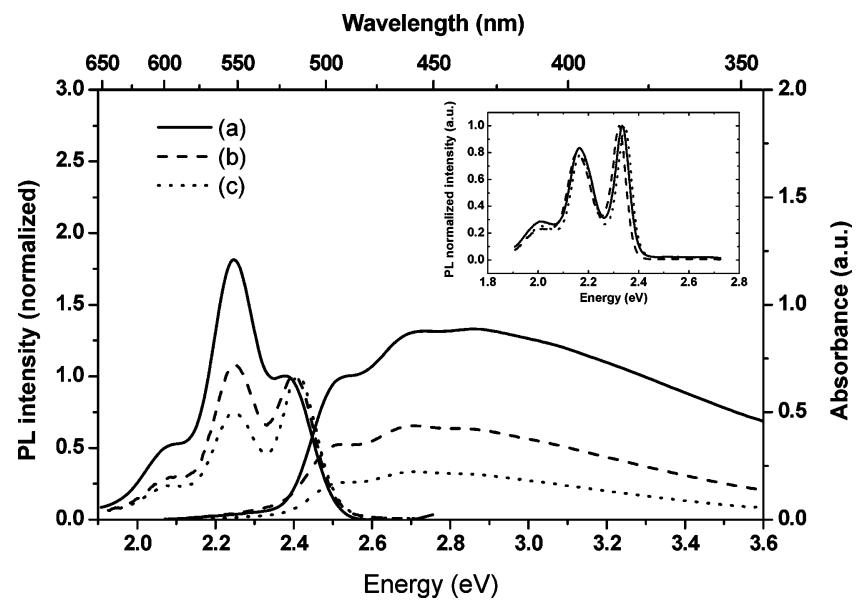

Figure 3. Room-temperature optical absorption and photoluminescence spectra of standard PPV films prepared with different diluted solutions of the polymer precursor. (a) Low (1:4), (b) medium (1:10), and (c) high dilution (1:20). In the inset, we present photoluminescence spectra observed at $87 \mathrm{~K}$ of the same PPV films prepared at $T_{\mathrm{c}}=300^{\circ} \mathrm{C}$ : (a) low dilution (1:4); (b) medium dilution (1:10), and (c) high dilution $(1: 20)$. All PL spectra are normalized to the maximum intensity of peak 2 and have been obtained with the excitation energy $E_{\text {exc }}=2.81 \mathrm{eV}$.

In Figure $3 a-c$ left side, we show the influence of the dilution of the precursor polymer solution on PL data of PPV samples, which exhibit significant changes in the relative intensity of peak 2 (at $\sim 2.43 \mathrm{eV}$ ) with respect to peak 1 (at $\sim 2.25 \mathrm{eV}$ ). This effect seems to indicate a decrease of the effective conjugation lengths when the precursor dilution increases. To provide further arguments in the model presented later, we give in the inset the PL spectra of PPV films obtained with different precursor polymer dilutions, recorded at $87 \mathrm{~K}$. All spectra shown there have the same features and present no change in their relative intensity.

3.3. Further Spectroscopic Characterization. To collect further structural information, in particular on the conjugation length distribution, Raman scattering data from the different samples are given in Figure 4. We show in Figure $4 a-c$ Raman scattering spectra of PPV in the $1100-1700 \mathrm{~cm}^{-1}$ range for the excitation wavelength $\lambda_{\mathrm{exc}}=1064 \mathrm{~nm}$ at different conversion temperatures. The main peaks (labeled 1-5) are located at 1174, $1330,1550,1586$, and $1625 \mathrm{~cm}^{-1}$ (see ref 26). The major change of these spectra is the increase of the peak intensity at 1625 $\mathrm{cm}^{-1}$ with respect to that of the peak at $1550 \mathrm{~cm}^{-1}$ for decreasing conversion temperature. ${ }^{13}$ This is the signature of the increase of the short segment contribution in the samples when the conversion temperature decreases. ${ }^{25}$

In Figure $4 \mathrm{~d}-\mathrm{f}$, we show the resonant Raman scattering spectra (RRS) taken with the excitation wavelength $\lambda_{\text {exc }}=363$ $\mathrm{nm}$ for the films prepared with three dilution concentrations of the precursor polymer solution in the $1100-1700 \mathrm{~cm}^{-1}$ range. As can be seen, all RRS spectra are characterized by the same five main bands, without frequency dispersion. These Raman bands are attributed mainly to stretchings and bendings of carbon bonds in the PPV molecular backbone. ${ }^{26} \mathrm{We}$ note that the relative intensity ratios $I_{1174 /} I_{1586}\left(I_{1} / I_{4}\right)$ and $I_{1550} / I_{1625}\left(I_{3} / I_{5}\right)$ as function of the dilution, given in the inset of Figure 4, decrease as the dilution increases. This trend is the consequence of an increase in the different films of the weight of the distribution of short conjugated segments with respect to that of the long segments. This behavior is similar to what is observed in different PPV films: (i) converted at different temperatures (see 


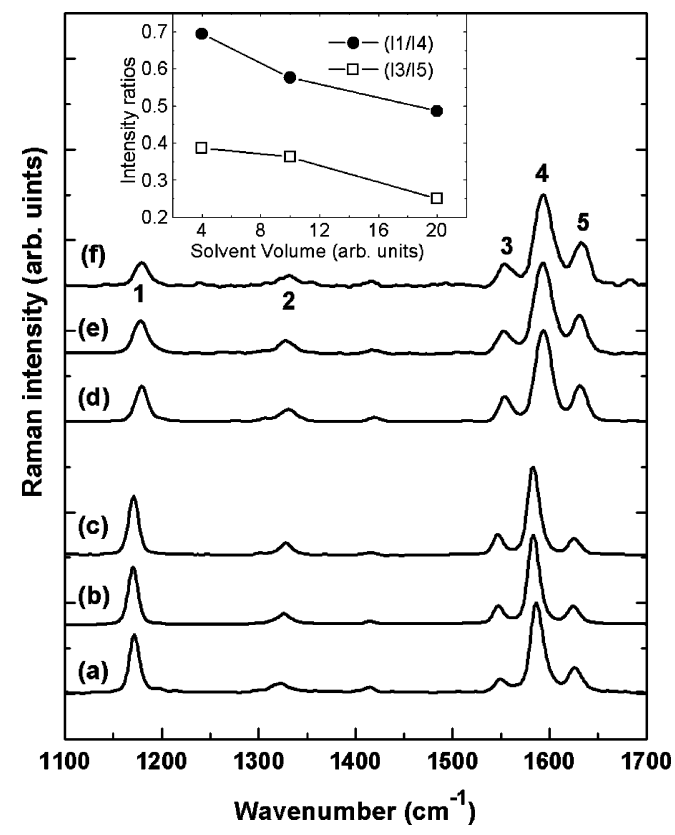

Figure 4. Room-temperature Raman scattering spectra recorded for an exciting wavelength $\lambda_{\text {exc }}=1064 \mathrm{~nm}$ of PPV films converted at different temperatures $T_{\mathrm{c}}$ : (a) $120^{\circ} \mathrm{C}$, (b) $180{ }^{\circ} \mathrm{C}$, and (c) $300{ }^{\circ} \mathrm{C}$ and room-temperature resonant Raman scattering spectra of PPV films for $\lambda_{\text {exc }}=363 \mathrm{~nm}$ prepared with different diluted solutions of the polymer precursor: (d) low (1:4); (e) medium (1:10), and (g) high dilution (1: $20)$. In the inset, we show the intensity ratios (ם) $I_{1} / I_{4}$ and (O) $I_{3} / I_{5}$ as a function of the polymer precursor solution volume. Raman spectra have been normalized to the maximum of the band around $1591 \mathrm{~cm}^{-1}$.

also ref 4), (ii) in PPV-SWNT composite films as the $x$ concentration increases, ${ }^{15}$ and (iii) in PPV oligomers of various lengths. ${ }^{13}$

To investigate the structural modification induced by the dilution increase of the precursor polymer solution, we have carried out X-ray diffraction (XRD) studies on the samples obtained with low 1:4 and high 1:20 dilution, respectively. Figure 5a presents the XRD diagram of a film prepared with $1: 4$ dilution of the precursor solution. One observes peaks centered at $2 \theta=22.5^{\circ}$ and $28.5^{\circ}$ with a shoulder at $2 \theta=20.7^{\circ}$. This XRD diagram is in good agreement with results obtained elsewhere ${ }^{4,27}$ in which a careful study on a stretched PPV film has been performed. Both peaks are characteristic of the crystalline phase of PPV, corresponding to (110/200) and (210) reflections, assuming that chains are aligned along the $c$ direction (parallel to the stretching direction of the PPV film) in a monoclinic unit cell. We can deduce that the film prepared with the 1:4 dilution contains crystallized domains with a average size of $1.2 \mathrm{~nm}$, estimated by using the Scherrer's formula. ${ }^{28}$ This result shows that the crystallized domains are smaller than those reported elsewhere. ${ }^{29,30}$ In Figure 5b, the XRD diagram is given for the 1:20 dilution. Note that the peak at $2 \theta=22.5^{\circ}$ is no longer recorded while the one at $2 \theta=28.5^{\circ}$ is still observable. This proves that few PPV chains are still present in the film obtained with a high dilution, but completely disorganized. This feature is different from that observed in composite films PPV-SWNT. ${ }^{17}$ In particular, at high SWNT concentration $x=64 \%$, the XRD pattern shows a peak at $19.5^{\circ}$, which implies a smaller coherence length with respect to the pristine polymer. ${ }^{15}$ Alternatively, the XRD data taken from samples obtained for $T_{\mathrm{c}}=300{ }^{\circ} \mathrm{C}$ gives diagrams that are very similar to that of Figure 5a. Only for the sample converted at $T_{\mathrm{c}}=120^{\circ} \mathrm{C}$, the XRD data are similar to those given in Figure $5 b$.
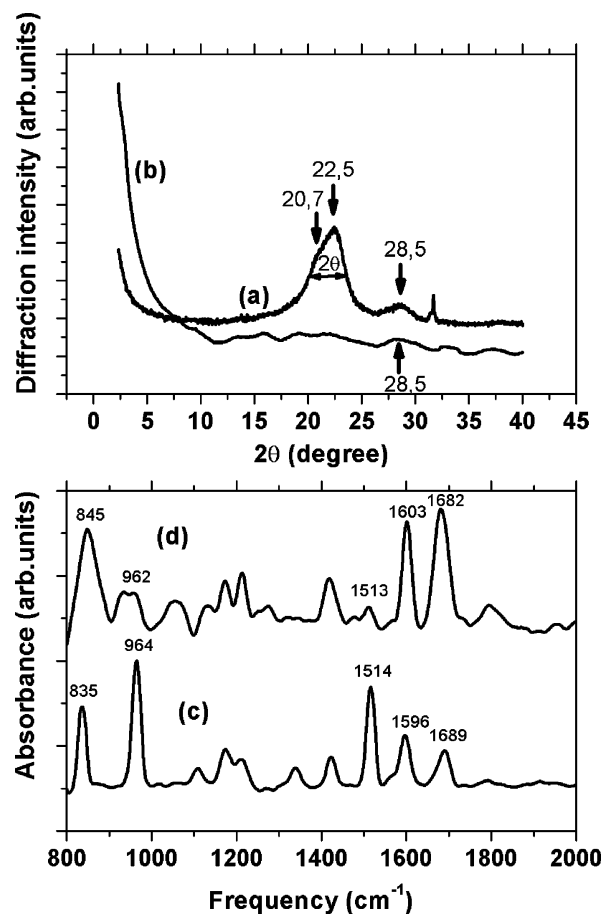

Figure 5. Top curves: X-ray diffraction patterns of two PPV films converted at $300{ }^{\circ} \mathrm{C}$ with: (a) low dilution of the precursor polymer solution (1:4) and (b) high dilution (1:20). Bottom curves: infrared spectra of two PPV films converted at $300{ }^{\circ} \mathrm{C}$ with (c) low dilution of the polymer precursor solution (1:4) and (d) high dilution (1:20).

Fourier-transform infrared absorption measurements were performed on two films obtained with a low and a high dilution, respectively. This has been done in order to eventually detect structural and chemical defects (Figure $5 \mathrm{c}$ and d), despite the difficulty to extract an exploitable signal from the latter film. Both IR spectra in Figure 5c and d are recorded in the 800$2000 \mathrm{~cm}^{-1}$ range. From the spectrum shown in Figure $5 \mathrm{~d}$ compared to that in Figure 5c, we observe a decrease of the two bands at $962 \mathrm{~cm}^{-1}\left(964 \mathrm{~cm}^{-1}\right)$, attributed to an out-ofplane bending vibration of the trans-vinyl group and at 1513 $\mathrm{cm}^{-1}\left(1514 \mathrm{~cm}^{-1}\right)$, characteristic of the stretching vibration of phenyl ring in "para" configuration. Moreover, we notice an increase of the two bands at $1603 \mathrm{~cm}^{-1}\left(1596 \mathrm{~cm}^{-1}\right)$ corresponding to symmetry defects on chains and at $1682 \mathrm{~cm}^{-1}(1689$ $\mathrm{cm}^{-1}$ ) characteristic of a stretching vibration of carbonyl groups. These changes agree very well with the study on the photooxidation of PPV films irradiated with a Xe lamp under ambient conditions in air. ${ }^{31}$ The relative intensity variation of these bands are quite consistent with the formation of chemical defects such as carbonyl groups $(\mathrm{C}=\mathrm{O})$, which disrupt the conjugation length of the segments in the chains of this film. Because of the large electron affinity of $\mathrm{C}=\mathrm{O}$, these defects can trap and dissociate excitons explaining, in part, the quenching of the PL as the dilution increases. We assume that these chemical defects stem from atmospheric oxygen diffusing from the film surface into the bulk and break the vinyl double bond of the polymer. Moreover, the less-organized structure of the film made with the high diluted solution could favor the diffusion rate of oxygen and, consequently, the rate at which damage occurs. ${ }^{32}$ FTIR data confirm the shortening of the effective conjugation length in the film obtained with high dilution of the precursor polymer by the presence of chemical defects as compared to standard PPV film.

We present in Figure 6 the spectrally integrated kinetics of the samples investigated by time-resolved photoluminescence spectroscopy. All signals are normalized to the maximum PL 


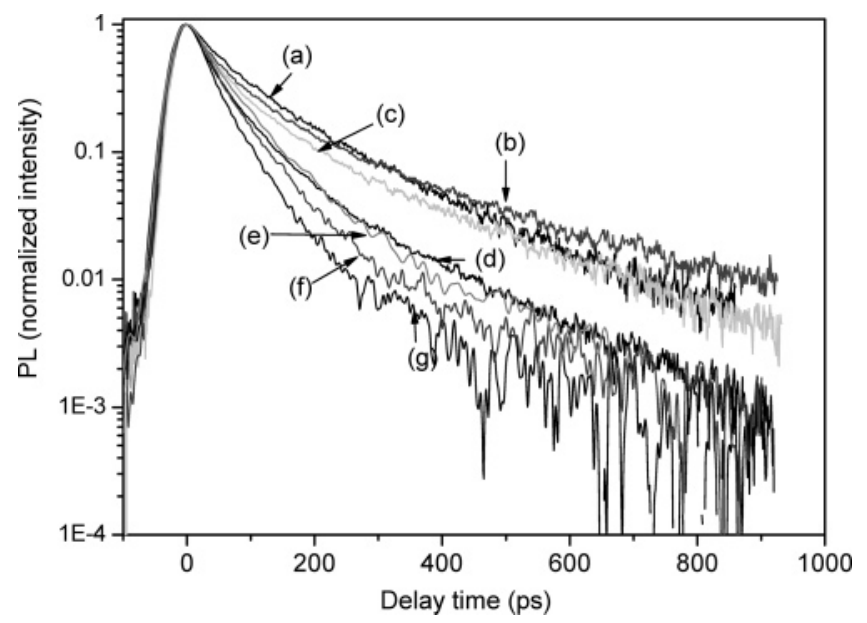

Figure 6. Transient photoluminescence kinetics recorded on PPV films converted at different temperatures, $T_{\mathrm{c}}$, prepared with different concentrations of the precursor solution and with different SWNT mass percentages $x$ as indicated: (a) $T_{\mathrm{c}}=300{ }^{\circ} \mathrm{C}$ and high diluted solution of the polymer precursor $(1: 20)$; (b) $T_{\mathrm{c}}=120^{\circ} \mathrm{C}$; (c) $T_{\mathrm{c}}=180^{\circ} \mathrm{C} ; T_{\mathrm{c}}$ $=300{ }^{\circ} \mathrm{C}$ for (d) low diluted solution (1:4) and for (e) $x=2 \%$, (f) $x$ $=16 \%,(\mathrm{~g}) x=32 \%$.

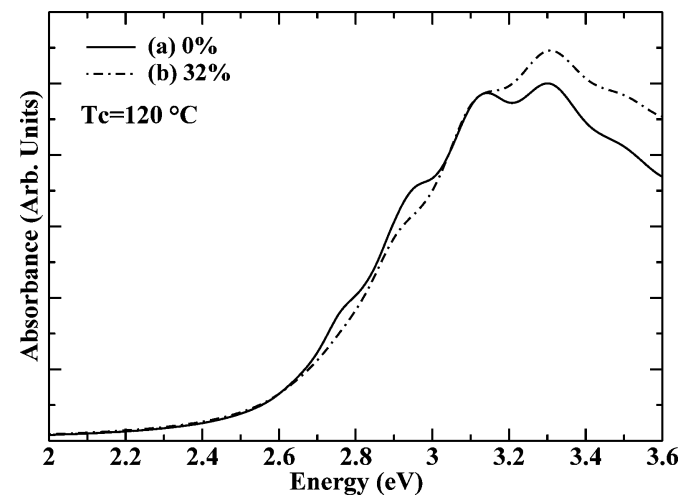

Figure 7. Calculated optical absorption at $300 \mathrm{~K}$ of the PPV-SWNT composite films obtained at the conversion temperature $T_{\mathrm{c}}=120{ }^{\circ} \mathrm{C}$ and for different SWNT mass percentages: (a) $x=0 \%$, (b) $x=32 \%$.

intensity and recorded in the $0-1000$ ps time range. The transient PL spectra (not shown here) are identical in energy to those obtained in steady-state PL. We observe that the PL decay rate varies drastically with the morphology of the samples, depending on several preparation conditions such as the precursor polymer dilution, conversion temperature, and SWNT concentration $x$. In particular, the recombination lifetime of PPV sample shown in Figure 6d (similar to that of standard PPV), is longer than that in samples containing a high SWNT concentration (Figure 6g), and shorter than that in samples characterized by high dilution (Figure 6a) and low conversion temperature of precursor polymer (Figure 6b). These results will be discussed later.

3.4. Theoretical Model and Band Shape Calculations. We show in Figure $7 \mathrm{a}$ and $\mathrm{b}$ the calculated optical absorption band shapes in the $2.00-3.60 \mathrm{eV}$ range for the two samples with $x$ $=0 \%$ and $32 \%$, as indicated, obtained at the conversion temperature $T_{\mathrm{c}}=120{ }^{\circ} \mathrm{C}$. We have used the model given in refs 13 and 14 and the same values for the absorption electronic energies and the electron vibration couplings given in Table 1 of ref 14. All of these values depend on $n$, which numbers the phenyl rings in the conjugated segments whose lengths are considered from $n=2-10: n=2-6$ refers to segments whose electronic transition energies depend on $n$ (short segments), while $n=7-10$ refers to those whose electronic transition
TABLE 1: Bimodal Distribution Parameters Used in the Evaluation of the Different Spectra Shown in the Figures as Indicated

\begin{tabular}{llllll}
\hline figures & $n_{1}$ & $\sigma_{1}$ & $n_{2}$ & $\sigma_{2}$ & $\mathrm{G}$ \\
\hline $7 \mathrm{a}, 8 \mathrm{~b}, 9 \mathrm{a}$ & 2 & 1 & 4 & 1 & 0.35 \\
$7 \mathrm{~b}, 9 \mathrm{c}$ & 2 & 1 & 4 & 2 & 0.23 \\
$10 \mathrm{a}, 11 \mathrm{a}$ & 3 & 1 & 6 & 1 & 0.30 \\
$10 \mathrm{~b}, 11 \mathrm{~b}$ & 3 & 1 & 6 & 1 & 0.25 \\
$10 \mathrm{c}, 11 \mathrm{c}$ & 3 & 1 & 6 & 1.2 & 0.20 \\
$8 \mathrm{a}$ & 3 & 1 & 5 & 0.9 & 0.22 \\
$9 \mathrm{~b}$ & 2 & 1 & 4 & 0.7 & 0.28
\end{tabular}

energies are independent of $n$ (long segments). In this model, the short segments are associated with the disordered regions, and the long segments with the well-packed regions of the samples. In Table 1 of the present paper, we give the bimodal distribution parameters used in these evaluations: $G$ is the weight of the longest segment distribution (centered on $n_{2}$ ) with respect to the shortest one (centered on $n_{1}$ ) in a given sample, and $\sigma_{1}$ and $\sigma_{2}$ are the standard deviations for the two distributions, respectively. Note that in this particular case the two distributions are centered on short segments and that the sum in the evaluations are always performed from $n=2$ to $n=10$.

The absorption band shapes and their structures shown in Figure $7 \mathrm{a}$ and $\mathrm{b}$ to be compared with those of Figure 2, are determined by the $\pi \rightarrow \pi^{*}$ electronic transitions of the segments that have more weight in the distribution. Furthermore, the relative intensity of the peak at $\sim 3.25 \mathrm{eV}$ with respect to the peak at $3.1 \mathrm{eV}$, going from $0 \%$ to $32 \%$, increases because of the increased weight of the very short segments in this latter case. In fact, the increase of the SWNT percentage in the composite films determines a further rise of the shorter segment contribution in the distribution, as it has been proved in ref 14 .

In Figure $8 \mathrm{a}$ and $\mathrm{b}$, we show the calculated Raman scattering spectra for $\lambda_{\text {exc }}=1064 \mathrm{~nm}$ obtained at $T_{\mathrm{c}}=180{ }^{\circ} \mathrm{C}$ and $T_{\mathrm{c}}=$ $120{ }^{\circ} \mathrm{C}$, respectively. Both spectra have been calculated by considering the model given in refs 13 and 15 and the electron vibration couplings given in ref 14 . In Table 1 , we give the values of the distribution parameters determined by the optical absorption band shapes. Note in these figures the changes of the relative intensity of the band peaked at 1550 and $1625 \mathrm{~cm}^{-1}$, going from the sample converted at $T_{\mathrm{c}}=180{ }^{\circ} \mathrm{C}$ to the one converted at $T_{\mathrm{c}}=120{ }^{\circ} \mathrm{C}$. The ratio $I_{1550} / I_{1625}$ in Figure $8 \mathrm{a}$ is nearly one, while in Figure $8 \mathrm{~b}$ it is less than one. This is a very important signature confirming that the short-segment contribution has more weight in the sample related to Figure $8 \mathrm{~b}$ and they are responsible for the preresonance condition at this excitation wavelength. Both spectra are in very good agreement with the experimental data shown in Figure $4 \mathrm{a}$ and $\mathrm{b}$.

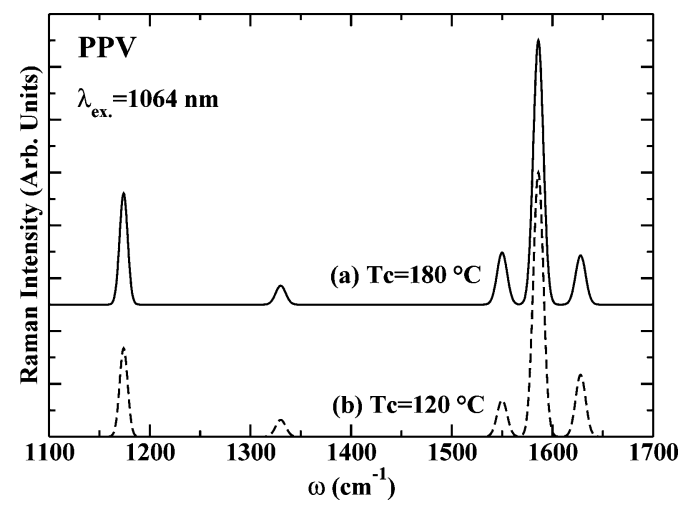

Figure 8. Calculated Raman spectra of PPV films converted at different temperatures as indicated: (a) $T_{\mathrm{c}}=180{ }^{\circ} \mathrm{C}$; (b) $T_{\mathrm{c}}=120{ }^{\circ} \mathrm{C}$; $\lambda_{\text {exc }}=$ $1064 \mathrm{~nm}$. 


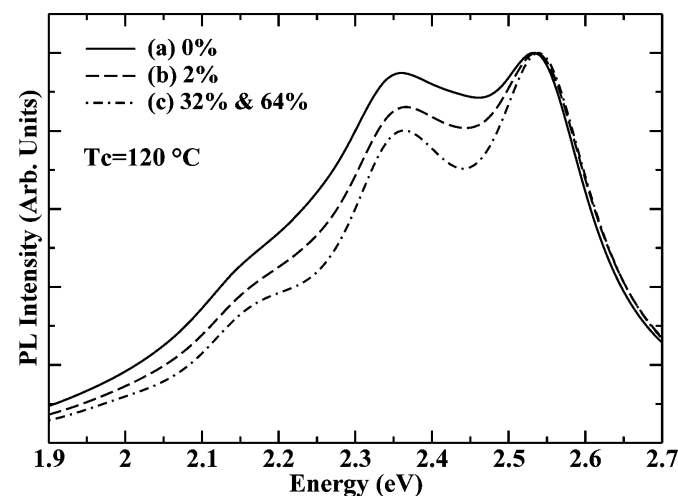

Figure 9. Calculated PL spectra at $300 \mathrm{~K}$ of PPV-SWNT composite films obtained at $T_{\mathrm{c}}=120{ }^{\circ} \mathrm{C}$, for $E_{\mathrm{exc}}=2.80 \mathrm{eV}$, and for different SWNT mass percentages: (a) $x=0 \%$, (b) $x=2 \%$, (c) $x=32 \%$ and $64 \%$. All of the bands are normalized to the peak at $2.55 \mathrm{eV}$.

TABLE 2: Values of Electronic Emission Energies $\boldsymbol{\Omega}_{n, e}$, Electron-Vibration Couplings $S_{f, n}$, and Emission Bandwidths $\gamma_{n, e}$ Used for the Calculations of Emission Band Shapes for Different Excitation Energies

\begin{tabular}{lcccccc}
\hline \multicolumn{1}{c}{$n$} & 2 & 3 & 4 & 5 & 6 & $7-10$ \\
\hline$\Omega_{n, e}(\mathrm{eV})$ & 3.51 & 2.82 & 2.54 & 2.44 & 2.31 & 2.26 \\
$S^{e}{ }_{1, n}$ & 0.14 & 0.14 & 0.15 & 0.16 & 0.16 & 0.16 \\
$S^{e}{ }_{2, n}$ & 0.06 & 0.06 & 0.06 & 0.06 & 0.06 & 0.06 \\
$S^{e}{ }_{3, n}$ & 0.07 & 0.07 & 0.08 & 0.10 & 0.11 & 0.12 \\
$S^{e}{ }_{4, n}$ & 0.78 & 0.62 & 0.57 & 0.50 & 0.42 & 0.34 \\
$S^{e}{ }_{5, n}$ & 0.15 & 0.11 & 0.11 & 0.10 & 0.10 & 0.07 \\
$\gamma_{n, e}(\mathrm{eV})$ & 0.05 & 0.05 & 0.06 & 0.06 & 0.07 & 0.07
\end{tabular}

In Figure $9 \mathrm{a}-\mathrm{c}$, we show the calculated PL spectra of PPVSWNT composite films obtained at $T_{\mathrm{c}}=120{ }^{\circ} \mathrm{C}$ at different concentration $x$ of SWNTs, as indicated. Calculations have been done by following the model given in refs 14 and 33 and by considering the bimodal distribution parameters obtained from the absorption data and given in Table 1. The values of the emission electronic energies and the electron vibrational interaction couplings for each conjugated segment of length $n$, entering the simulation are reported in Table 2. In this particular case, the calculated PL spectrum is determined by the contributions to the band shape coming from the emission transitions of $n=$ 4 up to $n=10$ conjugated segments, weighted by the bimodal distribution. In the proposed model, we are able to account for the contributions of the emission transitions from disordered regions (short segments for $n=4-6$ ) and from "well-packed" regions (long segments $n=7-10$ ). In the spectrum of Figure 9a, the band peaked at $\sim 2.55 \mathrm{eV}$ (peak 4) is due to the $0-0$ electronic emission transition ${ }^{34}$ of $n=4$ conjugated segments. The band peaked at $\sim 2.36 \mathrm{eV}$ is determined mainly by the $0-0$ emission transition of the $n=6$ segments and by the first and second-order vibronic replicas of the $n=4$ and $n=5$ pure $0-0$ electronic transitions. The latter one at $\sim 2.43 \mathrm{eV}(n=5$ electronic transition), hidden in the broad band, is responsible of the asymmetric shape of the band peaked at $2.36 \mathrm{eV}$. The 0-0 emission transitions of 7-10 long segments at $2.23 \mathrm{eV}$ are responsible of the broad shape together with the vibronic replicas of shorter segments in the frequency region 2.25-2.10 $\mathrm{eV}$, with a small bump at $2.16 \mathrm{eV}$. By increasing the $x$ concentration in the composite films, the calculated PL spectra change as shown in Figure $9 b$ and $c$. The relative intensity increase of the peak at $2.55 \mathrm{eV}$ with respect to the peak at 2.36 $\mathrm{eV}$, is accounted for by the increased weight of the $n=4$ segments, with respect to those with longer conjugation lengths.

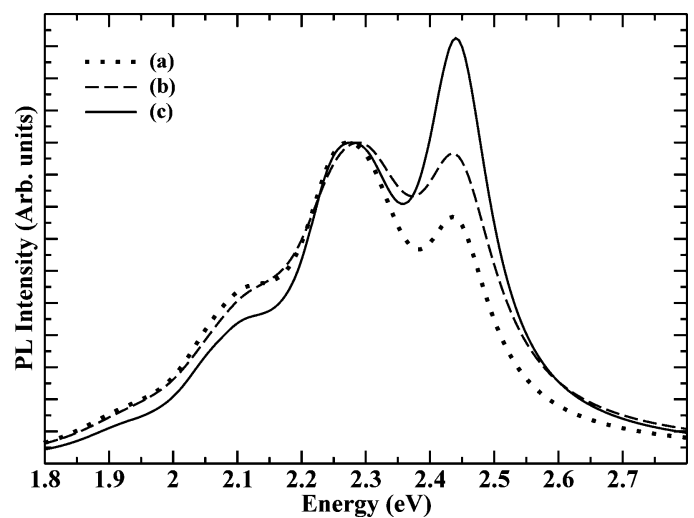

Figure 10. Calculated PL spectra of PPV films prepared with different diluted solutions of the polymer precursor and for $\mathrm{E}_{\mathrm{exc}}=2.80 \mathrm{eV}$ : (a) low (1:4); (b) medium (1:10); (c) high dilution (1:20). The bands are normalized to $2.25 \mathrm{eV}$ peak.

All of these spectra are in good agreement with the experimental data of Figure 2.

In Figure $10 \mathrm{a}-\mathrm{c}$, the calculated PL spectra for the PPV samples obtained from different diluted solutions are given. The evaluation is performed by using the distribution parameters of Table 1 and the electronic energy values given in Table 2 (see captions). Because the excitation energy is $E_{\mathrm{exc}}=2.81 \mathrm{eV}$, we consider the contributions to the band shapes from the segments whose conjugation lengths vary from $n=5$ up to $n=10$. In Figure $10 \mathrm{a}$, the PL spectrum is similar to the usual spectrum of standard PPV at the observation temperature $T=300 \mathrm{~K}$, as reported in refs 14, 33, and 35, with peak 1 at $2.25 \mathrm{eV}$, determined by 7-10 long segments ( $0-0$ transitions), peak 2 at $2.43 \mathrm{eV}$, determined by $n=5$ segments $(0-0$ transition), and a small bump at $2.10 \mathrm{eV}$, due to the vibronic replicas of the previous transitions. In the calculated spectra shown in Figure 10b and c for the samples prepared with an increased dilution of the solution, the weight of the isolated short segments with $n=5$ rises with respect to the well-packed regions as shown in Table 1. In this way, the increase of the relative intensity of peak 2 with respect to peak 1 is accounted for (Figure 10), in very good agreement with experimental data given in Figure 3. The interpretation of the changes of the PL spectra (Figure 10) in terms of the increase of the short and isolated segments is consistent with the X-ray diffraction data given in Figure 5b. It is observed that for the samples prepared from highly diluted solutions, the peak at $2 \theta=22.5^{\circ}$, due to crystallized domains disappears, while the peak at $2 \theta=28.5^{\circ}$ is still present. This peak is the signature that PPV conjugated segments present in the sample are responsible for the disordered regions with short and isolated segments, while well-packed regions are almost absent.

The evaluation of the Raman band shapes are given in Figure 11. They are obtained by using the bimodal distribution parameters in Table 1, deduced from the absorption band shape, and the model reported in refs 13 and 15. Note that from Figure 11 one can deduce that the intensity ratios $\left(I_{1} / I_{4}\right.$ and $\left.I_{3} / I_{5}\right)$ decrease as function of the dilution, as a consequence of the increase of the short and isolated segments (see Table 1) and in agreement with the experimental data given in the inset of Figure 4. This trend is the consequence of the increased contribution of the short segment vibrational frequencies in the Raman scattering band-shape intensities, as it has been reported previously in refs 13 and 15. The results given in Figure 11 are in good agreement with the experimental data of Figure $4 \mathrm{~d}$ and $\mathrm{f}$. 


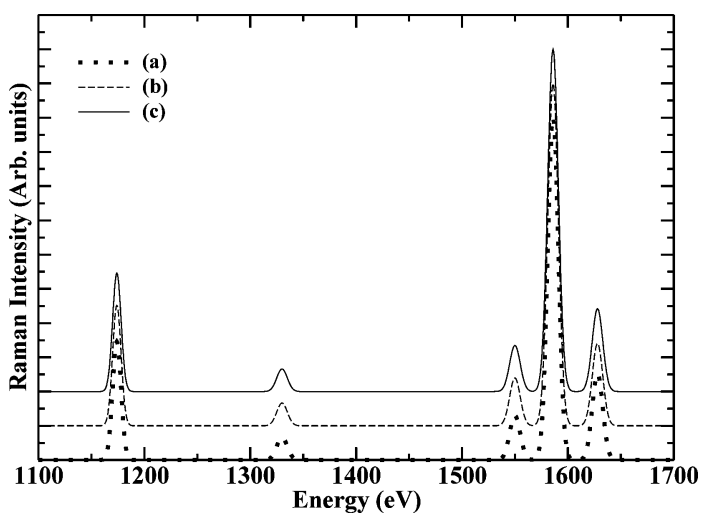

Figure 11. Calculated resonance Raman scattering spectra of PPV films at $300 \mathrm{~K}$ prepared at $T_{\mathrm{c}}=300{ }^{\circ} \mathrm{C}$ with different diluted solutions for $\lambda_{\mathrm{exc}}=363 \mathrm{~nm}$ : (a) low (1:4); (b) medium (1:10); (c) high dilution $(1: 20)$

\section{Discussion and Conclusion}

The experimental data presented here are interpreted in the frame of the bimodal distribution of effective lengths of PPV conjugated segments whose theoretical analysis is also presented. This theoretical model can be regarded as a morphological simulation of a polymer consisting of short isolated segments (disordered regions) and well-packed quasi-crystalline long segments. We recall that in the model proposed the $n=2-6$ and $n=7-10$ segments refer to isolated segments in the disordered regions and to the long segments that simulate the well-packed regions, respectively. Therefore, by using this model we can account for a bimodal inhomogeneity in the polymer, also in agreement with the results presented in refs 16 and 36. Let us recall that the bimodal distribution of conjugated segments has been introduced in PPV already for the interpretation of absorption and Raman data, ${ }^{13}$ but since then has been put in evidence in other polymers such as polydithienylmethine. ${ }^{37}$

Absorption, Raman, and PL spectra are very well reproduced by the calculations based on short- and long-segment distribution and their electronic and vibrational properties. In Figures 2 and 9 , the PL spectra are given for the samples converted at $T_{\mathrm{c}}=$ $120{ }^{\circ} \mathrm{C}$ as function of the SWNT $x$ concentration. We have also shown in Figure 3 and Figure 10 that PL spectra change as a function of the dilution of the precursor solution. From the inset of Figure 3, one can derive that they change as well as with the observation temperature from 300 to $87 \mathrm{~K}$. As a matter of fact, the interpretation of the PL spectra needs to consider the migration of the excitations from short to long segments. This is a temperature-dependent effect, contrary to the energy-transfer Förster mechanism, which is temperature-independent. Such a mechanism has already been discussed intensively in ref 35 . As one can see at room temperature, the contribution of short segments (peak 2 of Figures 3 and 10) increases dramatically in the film prepared from high diluted solution with respect to that of peak 1 becausr of the long segments. On the contrary, peak 1 is the predominant one in the PL spectra of samples prepared with low diluted solution. At $87 \mathrm{~K}$, no difference in PL spectra of all samples is observed. This can be explained only by accounting for a temperature-dependent migration mechanism from short to long segments (see refs 14, 33, and 35). This conclusion is also corroborated by considering the PL decay given in Figure 4 of ref 18 where it is shown that the PL decay rate of PPV depends strongly on temperature while that of PPV-69\%polyvinylalcohol does not show dependence up to $1 \mathrm{~ns}$. Moreover, the data in ref 38 show a strong temperature dependence of the PPV fluorescence decay from 290 to $16 \mathrm{~K}$, which in addition is similar to that of 1,4-bis((4',4"'-bisstyryl)styrylbenzene):polystyrene (OPV5:PS). These results imply that while at room temperature the PL spectrum of standard PPV is dominated by the contribution of long segments, at low temperature it is characterized by recombination on short segments..$^{35}$

Furthermore, the PL decay can give deep insight into the sample morphology (see Figure 6 curves a-d) which can be checked also from XRD data (Figure 5a and b). In Figure 6, it is shown that the recombination rate in the standard PPV sample, when the long segments (well-packed regions) are predominant (Figure 6d), is faster than that in the sample obtained from high dilution (Figure 6a) characterized by isolated and short segments. In agreement with this interpretation, one can derive from XRD data that in standard PPV the well-packed and ordered regions are responsible for the diffraction peak at $2 \theta=22.5^{\circ}$, which disappears totally in the high-dilution sample. Alternatively, from room-temperature steady-state PL spectra of samples obtained with $T_{\mathrm{c}}=120{ }^{\circ} \mathrm{C}$ (Figure 2), one can deduce that the most-intense feature of the spectra is the peak 4 at $2.55 \mathrm{eV}$, due to the recombination on $n=4$ segments, with respect to the peak due to the $n=6$ segments. The peak due to the $n=$ 6 segments decreases in intensity when SWNTs are added in the prepolymer solution, while the contribution of the long segments to the peak at $2.23 \mathrm{eV}$ is almost negligible. The decay rate observed in the transient PL (Figure 6b), which is similar to that of high-dilution samples (Figure 6a), corroborates this interpretation. Also, the XRD data (not shown here) of the sample prepared at $T_{\mathrm{c}}=120{ }^{\circ} \mathrm{C}$ exhibit diffraction patterns similar to that of Figure $5 \mathrm{~b}$ for high-dilution samples, proving that well-packed regions are almost absent in the samples. This further corroborates the morphological picture we have presented. A different situation occurs in the PPV-SWNT composite films for which the transient data given in Figure $6 e-g$ shows a faster decay with respect to that of Figure $6 \mathrm{~d}$ (standard PPV). In these cases, we have to take into account both the shortening of the conjugated segments and the competition with the increase of photoconductivity as discussed in ref 15. This nonradiative process is responsible of the PL quenching at room temperature. The main contribution to the PL decay originates from the recombination of charges on the short segments where they are created. Alternatively, the nanotubes can constitute a network for the charges that can migrate from short to longer segments. From the XRD of PPVSWNT with $x=64 \%$ given in ref 17 , it is shown that there is a peak at $2 \theta=19.5^{\circ}$ corresponding to the PPV regions with shorter coherence length together with a peak at $2 \theta=26.1^{\circ}$ related to SWNT bundles. The presence of bundles and still well-packed regions in the composite films can support the interpretation given above.

The experimental and theoretical spectroscopic investigation on the polymeric compounds we have presented in this paper gives important information on the distribution of conjugation lengths, which depend to a larger extent on the content of chemical and conformational defects in the polymeric chains. As a matter of fact, the conjugation lengths are usually significantly shorter than the chain length. Therefore, SEC (size exclusion chromatography) cannot provide any insight into this problem because the molecular weight distribution given by this kind of measurement is not related in a straightforward manner to the distribution of conjugation lengths.

In summary, a detailed analysis of the optical properties of several PPV samples provides a better understanding of the role 
of the different parameters influencing the synthesis of this polymer. These include the conversion temperature, the dilution concentration of the pre-polymer solution, and the SWNT $x$ concentration in composites. All data are well interpreted by considering a bimodal distribution of conjugated segments.

Acknowledgment. We thank Dr. R. Almairac from Laboratoire des Colloïdes, Verres et Nanomatériaux (Unité Mixte de Recherche CNRS-Université de Montpellier II N 5587) for his help in X-ray diffraction studies and Dr. Adam Pron, from CEA-Grenoble for discussion concerning SEC data. E.M. acknowledges financial support from EU's Framework Programme through the Nanoquanta Network of Excellence (NM P4-CT-2004-500198). The Institut des Matériaux Jean Rouxel is Unité Mixte de Recherche $\mathrm{n}^{\circ} 6502$ CNRS-Université de Nantes.

\section{References and Notes}

(1) Friend, R. H.; Gymer, R. W.; Holmes, A. B.; Burroughes, J. H.; Marks, R. N.; Taliani, C.; Bradley, D. D. C.; Santos, D. A. D.; Bredas, J. L.; Logdlund, M.; Salaneck, W. R. Nature 1999, 397, 121.

(2) Burroughes, J. H.; Bradley, D. D. C.; Brown, A. R.; Marks, R. N.; Mackay, K.; Friend, R. H.; Burn, P. L.; Holmes, A. B. Nature 1990, 347, 539.

(3) Gustafsson, G.; Gao, Y.; Treacy, G. M.; Klavener, F.; Colinari, N.; Heeger, A. J. Nature 1992, 357, 477.

(4) Wéry, J.; Dulieu, B.; Bullot, J.; Baïtoul, M.; Deniard, P.; Buisson, J. P. Polymer 1999, 40, 519.

(5) Burn, P. L.; Bradley, D. D. C.; Brown, A. R.; Friend, R. H.; Holmes, A. B. Synth. Met. 1992, 41-43, 261.

(6) Shah, H. V.; Scheinbeim, J. I.; Arbuckle, G. A. J. Polym. Sci. Part B: Polym. Phys. 1999, 37, 605.

(7) Köpping-Grem, G.; Leising, G.; Schimetta, M.; Stelzer, F.; Huber, A. Synth. Met. 1996, 76, 53. 295.

(8) Bjorklund, T. G.; Lim, S. H.; Bardeen, C. J. Synth. Met. 2002, 126,

(9) Nguyen, T. Q.; Doan, V.; Schwartz, B. J. J. Chem. Phys. 1999, $110,4068$.

(10) Shah, H. V.; Arbuckle, G. A. Macromolecules 1999, 32, 1413.

(11) Papadimitrakopoulos, F.; Konstadinidis, K.; Miller, T. M.; Opila, R.; Chandross, E. A.; Galvin, M. E. Chem. Mater. 1994, 6, 1563.

(12) Morgado, J.; Cacialli, F.; Gruner, J.; Greenham, N. C.; Friend, R. H. J. Appl. Phys. 1999, 85, 1784.

(13) Mulazzi, E.; Ripamonti, A.; Wéry, J.; Dulieu, B.; Lefrant, S. Phys. Rev. B 1999, 60, 16519.

(14) Wéry, J.; Aarab, H.; Lefrant, S.; Faulques, E.; Mulazzi, E.; Perego, R. Phys. Rev. B 2003, 67, 115202.
(15) Mulazzi, E.; Perego, R.; Aarab, H.; Mihut, L.; Lefrant, S.; Faulques, E.; Wéry, J. Phys. Rev. B 2004, 70, 155206.

(16) Collison, C. J.; Tremaneekam, V.; Oldham, W. J.; Tsu, J. H.; Tothberg, J. L. Synth. Met. 2001, 119, 515.

(17) Aarab, H.; Baïtoul, M.; Wéry, J.; Almairac, R.; Lefrant, S.; Faulques, E.; Duvail, J. L.; Hamedoun, M. Synth. Met. 2005, 155, 63.

(18) Bjorklund, T. G.; Lim, S.-H.; Bardeen, C. J. Synth. Met. 2004, 142, 195.

(19) Stenger-Smith, J. D; Lenz, R. W.; Wegner, G. Polymer 1989, 30, 1048.

(20) Wéry, J.; Dulieu, B.; Launay, E.; Bullot, J.; Baïtoul, M.; Buisson, J. P. Synth. Met. 1997, 84, 277.

(21) Schlenoff, J. B.; Wang, L. J. Macromolecules 1991, 24, 6653.

(22) Woo, H. S.; Lhost, O.; Graham, S. C.; Bradley, D. D. C.; Friend, R. H.; Quattrocchi, C.; Bredas, J. L.; Schenk, R.; K. Müllen, K. Synth. Met. 1993, 59, 13.

(23) in het Panhuis, M.; Maiti, A.; Dalton, A. B.; van den Noort, A.; Coleman, J. N.; McCarthy, B.; Blau, W. J. J. Phys. Chem. B 2003, 107, 478.

(24) Cornil, J.; Heeger, A. J.; Bredas, J. L. Chem. Phys. Lett. 1997, $272,463$.

(25) Mulazzi, E.; Perego, R.; Aarab, H.; Faulques, E.; Lefrant, S.; Wéry, J. Synth. Met. 2005, 154, 221.

(26) Orion, I.; Buisson, J. P.; Lefrant, S. Phys. Rev. B 1998, 57, 7050.

(27) Moon, Y. B.; Rughooputh, S. D. D. V.; Heeger, A. J.; Patil, A. O.; Wudl, F. Synth. Met. 1989, 29, E79.

(28) Klug, H. P.; Alexander, L. E. X-ray Diffraction Procedures For Polycrystalline and Amorphous Materials; Wiley, New York, 1954.

(29) Chen, D.; Winokur, M. J.; Masse, M. A.; Karasz, F. E. Phys. Rev B 1990, 41, 6759 .

(30) Gagnon, D. R.; Karasz, F. E.; Thomas, E. L.; Lenz, R. W. Synth. Met. 1987, 20, 85.

(31) Rothberg, L. J.; Yan, M.; Papadimitrakopoulos, F.; Galvin, M. E.; Kwock, E. W.; Miller, T. M. Synth. Met. 1996, 80, 41.

(32) Nguyen, T. Q.; Martini, I. B.; Liu, J.; Schwartz, B. J. J. Phys. Chem. B 2000, 104, 237.

(33) Mulazzi, E.; Botta, C.; Facchinetti, D.; Bolognesi, A. Synth. Met. 2004, 142, 85 .

(34) By 0-0 electronic transition, we mean pure electronic transition from the lowest excited state to the lowest ground state, for each conjugated segment. The energy of these transitions are given in Table 2.

(35) Mulazzi, E.; Perego, R.; Wéry, J.; Mihut, L.; Lefrant, S.; Faulques, E. J. Chem. Phys. 2006, 125, 014703.

(36) Comoretto, D.; Soci, C.; Marabelli, F.; Mikhailovsky, A.; Moses, D. Synth. Met. 2005, 153, 281.

(37) Curran, S. A.; Lim, Z. D.; Dundigal, S.; Blau, W. J. Phys. Chem. B 2006, 110, 3924 .

(38) Bjorklund, T. G.; Lim, S.-H.; Bardeen, C. J. J. Phys. Chem. B 2001, 48,11970 . 